\title{
mencente
}

Revista Educación

ISSN: 0379-7082

ISSN: 2215-2644

revedu@gmail.com

Universidad de Costa Rica

Costa Rica

\section{Diagnóstico para nueva oferta educativa a distancia: estudio de caso basado en expectativas educativas}

Luévano De la Cruz, Karla Olimpia; López Betancourt, Alicia; Orrante Reyes, María del Carmen

Diagnóstico para nueva oferta educativa a distancia: estudio de caso basado en expectativas educativas

Revista Educación, vol. 44, núm. 2, 2020

Universidad de Costa Rica, Costa Rica

Disponible en: http://www.redalyc.org/articulo.oa?id=44062184019

DOI: https://doi.org/10.15517/revedu.v44i2.39330

Esta obra está bajo una Licencia Creative Commons Atribución-NoComercial-SinDerivar 3.0 Internacional. 


\title{
Diagnóstico para nueva oferta educativa a distancia: estudio de caso basado en expectativas educativas
}

\author{
Assessing New Types of Distance Learning Programs: A Case Study Based on Educational Expectations \\ Karla Olimpia Luévano De la Cruz \\ Universidad Juárez del Estado de Durango, México \\ DOI: https://doi.org/10.15517/revedu.v44i2.39330 \\ Redalyc: http://www.redalyc.org/articulo.oa?id=44062184019
}

karlaluevano@ujed.mx

iD http://orcid.org/0000-0002-1912-114X

Alicia López Betancourt

Universidad Juárez del Estado de Durango, México

ablopez@ujed.mx

(iD http://orcid.org/0000-0002-3447-2503

Maria del Carmen Orrante Reyes

Universidad Juárez del Estado de Durango, México

maricarmen_orrante@hotmail.com

Recepción: 19 Diciembre 2019

Aprobación: 29 Mayo 2020

\section{RESUMEN:}

El Programa de Fortalecimiento para la Calidad Educativa (PFCE) es una iniciativa del gobierno de México para apoyar a las Universidades Públicas con recursos extraordinarios. La presente investigación apoyada por PFCE tuvo como objetivo principal generar un diagnóstico para nueva oferta educativa a distancia con el propósito de reducir la migración de los jóvenes a la capital, a otros estados o países. Para lo cual se determinaron los principales factores que influyen en la perspectiva educativa de estudiantes de educación media superior, en tres municipios de Durango, bajo la mirada de tres agentes informativos: la visión del alumnado, respecto a sus necesidades personales, académicas, familiares y sociales, la posición de sus madres y padres de familia respecto al futuro académico de sus hijas e hijos, así como las condiciones económicas y sociales en su contexto y por último, la perspectiva de los empleadores de la región con base en su experiencia y necesidades actuales. Sigue una metodología de estudio de caso de carácter mixto y en sentido transversal. Se aplicaron tres instrumentos y la información se procesó en SPSS, grupos de enfoque y entrevistas. Los resultados mostraron preferencias en: Ciencias de la Salud, Ciencias Sociales, Tecnología, Ciencias Naturales y Exactas, Geología, Agricultura, Educación y Emprendedurismo. Se recomienda ampliar y fortalecer la conectividad en los tres municipios para el buen funcionamiento de la educación a distancia. Así como un programa de educación continua para personas adultas y capacitación empresarial. El estudio resulta relevante porque precisa la oferta educativa contextualizada en las zonas de estudio para contribuir, en el mediano y largo plazo, al desarrollo social y económico de los municipios.

Palabras clave: Crecimiento económico, Desarrollo social, Educación, Educación a distancia, Perspectivas educativas.

\section{Abstract:}

The Program for Strengthening Educational Quality (PFCE) is an initiative of the Government of Mexico to support State Universities through the extension of additional resources. The main objective of this PFCE-supported study, was to assess a new distance learning program option to help reduce migration of youngsters to the capital city, other states or abroad. It was determined that the main factors that impact educational perspectives of high school students in three cities in the state of Durango, was based on three sources: The individual opinion of the student regarding their personal, academic, family and social needs, their parents' points of view about their children's academic future, varying economic and social contexts, and, lastly, current needs and perspectives of the region's employers based on their own experience. This case study is based on a crosscutting methodology through three surveys. The collected data was processed through SPSS, focus groups and interviews. The results reveal that students were particularly interested in pursuing Health and Social Sciences, Technology, Natural and Exact Sciences, Geology, Agriculture, Education and Business in a distance setting. Recommendations were made that the three cities that participated in the study make efforts to expand and enhance Internet connectivity in order to favor distance learning options, which also include Continuing Education Programs for adults, particularly Business Training Programs. This study is relevant 
since it aims to provide contextualized educational programs for specific fields of study and, therefore, help contribute to social and economic development of the region in the medium and long-term.

KeYwords: Economic Growth, Social Development, Education, Distance Learning, Educational Perspective.

\section{INTRODUCCIÓN}

Desde su nacimiento el ser humano tiende a buscar la sobrevivencia, el desarrollo y la realización personal; para lograrlo, interactúa con su entorno y socialmente, la familia es considerada la responsable de satisfacer estas necesidades. Por ello, se espera que la sociedad pueda satisfacer casi totalmente sus necesidades biológicas, sociales, culturales y económicas (Galeana, 2005). Ambas instituciones, familia y sociedad influyen de manera determinante en la manera y alcances del cumplimiento de estas necesidades.

La educación genera las condiciones de desarrollo social y económico que impulsan las economías locales y contribuyen a reducir las brechas de pobreza y desigualdad; es el catalizador más efectivo para desarrollar, potencializar y transmitir las habilidades, conocimientos y valores éticos de las personas, fundamental en los procesos de integración social, económica y cultural.

Con la intención de contribuir a la calidad educativa del país, el gobierno de México a través de la Secretaría de Educación Pública, otorga un presupuesto financiero extraordinario a nivel nacional mediante el Programa de Fortalecimiento a la Calidad Educativa, al cual todas las universidades pueden acceder mediante un concurso. Por su parte, la Universidad Juárez del Estado de Durango, (UJED), en el año 2016 recibió un recurso de este presupuesto, lo cual permitió cumplir con el propósito del presente estudio que fue elaborar un diagnóstico de las perspectivas para ofertar nuevas opciones educativas en modalidad a distancia en tres municipios específicos del estado, seleccionados por la propia Universidad para su análisis, siendo Santiago Papasquiaro, Tepehuanes y El Oro, los asignados para dicho proyecto. Para ello, se consideró la participación de tres agentes informativos; estudiantes que estuvieran cursando el tercer año del nivel medio superior, sus madres, padres o personas tutoras y como tercer agente, los empleadores de la región, en las cabeceras municipales, para indagar e identificar la situación demográfica, social y económica que tiene el alumnado del tercer año de educación media superior para determinar los factores que influyen en ellos para continuar con su educación superior, así como aquellos factores que obstaculizan dicho propósito, en relación con las perspectivas educativas de los tres agentes y con la oferta educativa actual.

A partir de lo anteriormente expuesto se deriva la siguiente pregunta: ¿Cómo pueden apoyar las expectativas del estudiantado, las personas progenitoras, contextos familiares y de empleabilidad a la apertura de nueva oferta educativa en modalidad a distancia? Para dar respuesta a la pregunta anterior, se determinaron las características de las expectativas educativas del alumnado, sus contextos familiares y de empleabilidad para que esta información dé sustento a la permanencia o creación de oferta educativa en modalidad a distancia en la UJED, a través del Sistema Universidad Virtual, en delante SUV, de esta Institución, pues se reconoce la importancia de la educación como potenciadora y desencadenante del crecimiento en diferentes vertientes como personal, social y económico.

La estructura del presente contiene los antecedentes, donde se hace una revisión cronológica resaltando los temas principales de la investigación. Seguida del marco teórico, donde se exponen las teorías que encaminan los conocimientos teóricos, posterior se encuentran los procedimientos metodológicos, donde se detalla la metodología de aplicación, así como el manejo de los resultados, por último, se presenta el análisis de los resultados, conclusiones y recomendaciones. 


\section{ANTECEDENTES}

A pesar de que el estado de Durango no se encuentra en los niveles más bajos de pobreza y educación a nivel nacional, las acciones tomadas en materia educativa permitirían fomentar el crecimiento económico del estado y sus municipios. En este sentido, el interés consiste en la elaboración de un diagnóstico basado en perspectivas de la ciudadanía del entorno, sobre las opciones para crear nueva oferta educativa del nivel superior en modalidad a distancia, que impulse el crecimiento de los municipios del estado y aunque es constante la mención del efecto de la educación en el progreso de una sociedad, la experiencia ha identificado una brecha sobre la forma en la cual la educación verdaderamente impacta el progreso de las regiones.

Específicamente para México se afirma que la inversión sostenida en educación, innovación y tecnologías de la información, conducirá al aumento en el uso y creación de conocimiento en la producción económica, dando lugar al crecimiento económico sostenido en todo al país (Sánchez y Ríos, 2011).

Con respecto a la relación de la educación con el mercado laboral Gabotto y Castellanos (2015) desarrollan una comparativa entre la situación laboral en México y la posibilidad de encontrar trabajo al egresar del nivel superior. Encuentran que tener un grado universitario no garantiza un empleo y que el tiempo de recuperación por la inversión en capital humano no se dará en el corto plazo. Por último, determinan que la población que demanda estudios superiores excede la oferta ofrecida. En consistencia con este desalentador panorama la visión de Márquez (2016) expresa que

para la mayor parte de la población mexicana, las perspectivas que les depara el futuro no son muy alentadoras: crisis económica, recortes presupuestales, crecimiento del empleo informal, incremento de la pobreza, etc. Pero esta realidad no la comparten todos los mexicanos en razón de la lacerante desigualdad en la distribución del ingreso económico. (p. 8)

Por el lado social, la Organización de las Naciones Unidas para la Educación, la Ciencia y la Cultura establece dentro de los Objetivos de Desarrollo Sostenible, el objetivo 4 que implica: "Garantizar una educación inclusiva, equitativa y de calidad y promover oportunidades de aprendizaje durante toda la vida para todos", (Organización de las Naciones Unidas para la Educación, la Ciencia y la Cultura, 2017, p.18). Este se retoma en la agenda 2030 al declarar a la educación transformadora de vidas, motor principal de desarrollo y clave para lograr el empleo y la erradicación de la pobreza. Esta agenda precisa la importancia del acceso a la educación, la inclusión y la equidad como piedra angular de una agenda transformadora, sustentada en igualdad de género; además, vela por educación de calidad y por la promoción de oportunidades de aprendizaje a lo largo de la vida para todos, sus contextos y niveles educativos.

Resulta prioritario que las instituciones de educación superior oferten y amplíen opciones de formación y actualización en las zonas rurales de México y tal como se efectúa esta investigación en zonas rurales del estado de Durango. Basados en la idea de que la educación permite a un individuo aumentar su nivel y calidad de vida, desarrollar pensamiento crítico reflexivo y propositivo, además, le permite contribuir a mejorar su entorno, estado y país.

Un factor detonante que motivó esta investigación fue reducir la migración de jóvenes a la capital, a otros estados o países, mediante nueva oferta educativa en el SUV de la UJED que les garantice recibir una preparación la cual impacte en su municipio y pueda impulsarlo al desarrollo social y económico.

Entonces resulta pertinente hacer estudios de caso, donde se pueda contextualizar la oferta educativa a la región y con ello garantizar en el mediano y largo plazo que los egresados tengan un empleo en su municipio, así como, un incremento en su calidad de vida y de la región donde se encuentran.

La educación a distancia viene a ser una posibilidad para lo anteriormente expuesto, la cual se ha globalizado con el advenimiento de la nueva tecnología, la Internet y la necesidad de contar con personal capacitado (Kumar, Kumar, Palvia y Verma, 2017). La economía global está cambiando, el área de los sistemas de información y la educación a distancia ofrecen flexibilidad a estudiantes y empleadores para el desarrollo educativo y profesional. La educación a distancia mejora la capacidad de las universidades para brindar 
educación a las áreas subatendidas. Vasilevska, Rivza, Pivac, Alekneviciene y Parlinska (2017) desarrollaron un estudio sobre la satisfacción en estudiantes y las fortalezas y debilidades de la educación a distancia en Europa del Este, en el cual, encontraron que la adaptabilidad, las capacidades técnicas, el conocimiento de la computadora, autocontrol y la motivación son habilidades importantes para el éxito de la educación en modalidad a distancia. Por su parte, Villafuerte y Benites (2018), destacan la metamorfosis que debe sufrir la educación tradicional hacia una educación de calidad y competencias que aporten a la economía el conocimiento, es decir, la generación de mano de obra calificada que pueda despuntar el crecimiento. De modo que la economía del conocimiento se verá cubierta por las instituciones educativas, aunque el conocimiento surja de ámbitos diversos, como empresas, instituciones científico-tecnológicas o políticas gubernamentales. Destacan dos hallazgos relevantes, uno es que el uso de las Tecnologías de la Información y de la Comunicación (TIC) resulta primordial para generar vínculos efectivos con la empresa y destacar su desempeño laboral y el otro es la coincidencia con Gabotto y Castellanos (2015) indicando que es el empleado quien debe adaptarse a las necesidades laborales que usualmente son cambiantes dependiendo de los giros y metas de la empresa.

\section{Marco teórico}

\subsection{Educación como potenciador de crecimiento económico y social}

Uno de los principales economistas, Adam Smith, plantea el esquema general de la Teoría del Crecimiento Económico, la cual se debe entender como un aumento sostenido de los estándares de vida de un espacio geográfico, tomando como unidad medida su capacidad productiva e ingresos. Como consecuencia de esto, es que surge el desarrollo social entendido como la mejora y crecimiento de las condiciones de vida de los integrantes de una sociedad.

El trabajo de Chaves (1997) puntualiza algunas relaciones entre economía y educación, señala que estos dos conceptos no pueden aislarse, sino por el contrario, cada uno de ellos funciona gracias al otro, la educación es materia de la economía y a su vez la educación es activadora de la economía. La educación aporta a la economía mediante el ingreso y la productividad y la economía aporta a la educación mediante la inversión en capital humano y la segmentación de los mercados. Además analiza la teoría del capital humano, considerando a la educación como un bien, en el cual se debe invertir, argumentando que mientras mayor sea la inversión mayores serán los rendimientos que podrán obtenerse, particularmente en una mejor distribución de los ingresos, sin embargo, señala que esta afirmación ha tenido dos resultados opositores, donde los partidarios a favor son estudios menos recientes que los partidarios en contra, es decir, mientras las tendencias del mundo globalizado aumentan, y el aporte que las industrias hacen a la producción, ciertamente se requiere mejor inversión en capital humano, pero no en la misma magnitud que hace décadas.

Por su parte, Bazdresch (2001) señala que en el contexto social, la importancia de la educación, entendida como escolarización, se fundamenta en la idea de que es la vía para alcanzar una vida mejor, es decir, para acceder a una posición económica y social más elevada. Esta conjetura sobre la función social de la educación es generalizada, pues se alude a la educación como un instrumento propulsor de la formación ciudadana y del desarrollo social y económico, así como de movilidad social; esto último, a través del vínculo que se establece entre educación, empleo e ingreso.

Ser incapaz de leer, escribir, o ejecutar las operaciones matemáticas básicas, e incluso no tener un nivel de escolaridad que la sociedad considera básico, limita las perspectivas culturales y económicas de todo ser humano, lo que restringe su capacidad para interactuar, tomar decisiones y funcionar activamente en su entorno social (Consejo Nacional de Evaluación de la Política de Desarrollo Social, 2009). 
Ante los avances tecnológicos a nivel mundial, el gobierno de México ha tenido la necesidad de actualizar los tradicionales métodos de enseñanza, en todos los niveles educativos, con el propósito de formar a las nuevas generaciones con herramientas y competencias que les permitan sobresalir en la nueva dinámica económica. Un ejemplo de estas acciones, se dio en el año 2015, cuando se implementó una política de intervención en la educación, denominada Programa de Inclusión Digital, orientada a estudiantes de 5to y 6to año de primaria, con el objetivo de reducir la brecha digital y el uso de las TIC en el proceso enseñanza aprendizaje, mejorando su calidad en la educación, siendo una de las metas del Plan Nacional de Desarrollo 2013-2018.

La aportación que tiene Durango al Producto interno bruto nacional es solo del 1.2\%, respecto al aporte estatal, el $59.1 \%$ proviene principalmente de actividades terciarias, el 31.0\% de actividades secundarias y el 9.9\% primarias, sin embargo es importante resaltar que Durango se encuentra entre los primeros lugares a nivel nacional en ciertos tipos de producción del sector primario, por ejemplo ocupa el primer lugar a nivel nacional en producción forestal de pino y encino, el tercero en extracción minera de plomo, plata y oro. Ocupa el segundo en producción forrajera y tercero en manzana y frijol y el primero en la producción de lobina.

La dinámica económica del país ha generado altas tasas de migración, tanto interna como externa, entendiéndose, que en la interna migran a otro estado del país y en la externa a otro país. A finales del año 2010, en México, el 22.0\% de población vivía en zonas rurales, cifra inferior a la que se presentaba en el estado Durango, pues para el mismo año, era del 31.0\% de la población estatal. En promedio en el municipio de Santiago Papasquiaro, el 8.5\% de la población proviene fuera del estado de Durango. En Tepehuanes el 3.7\% y en El Oro un 8.8\%.

\subsection{Educación como derecho social}

En México se ha establecido que recibir una educación de calidad a lo largo de toda la vida es un derecho innato de cada niño/niña, mujer y hombre (Organización de las Naciones Unidas para la Educación, la Ciencia y la Cultura, 2012); a razón de ello ha expresado su compromiso de hacer realidad el derecho que tiene cada persona a recibir enseñanza, pues se asume que la educación desempeña una función esencial en el desarrollo humano, social y económico.

En el marco normativo mexicano, el Artículo $3^{\circ}$ de la Constitución Política de los Estados Unidos Mexicanos y los artículos $2^{\circ}, 3^{\circ}$ y $4^{\circ}$ de la Ley General de Educación, establecen que toda la población debe cursar la educación preescolar, primaria y secundaria, niveles que constituyen la educación básica obligatoria y en su Artículo 31 aunado al Artículo $4^{\circ}$ de la Ley General de Educación señalan, la obligación del pueblo mexicano de enviar a sus hijas e hijos a las escuelas públicas o privadas para obtener la educación obligatoria.

En México, la educación media superior, complementa la enseñanza primaria superior, la cual capacita a sus estudiantes para vivir útilmente en la Nación y prepara al alumnado para el ingreso a la Universidad. La edad habitual del estudiantado de este nivel oscila entre 15 y 17 años (Instituto Nacional para la Evaluación de la Educación, 2011; Secretaría de Educación Pública, 2012). La educación media superior está compuesta por el bachillerato general, bachillerato tecnológico y profesional técnico, mezclándose a veces alguno de ellos con una duración promedio de tres años (Secretaría de Educación Pública, 2012).

Respecto al nivel de escolaridad, en México por cada 100 estudiantes de 15 años y más, solo 18.2, de ellos terminan el nivel medio superior, mientras que 14.6 son los que terminan el nivel superior. La gran mayoría logra terminar la educación básica, que en México incluye primaria y secundaria (Instituto Nacional de Estadística y Geografía, 2017). En la Tabla 1 se observa la situación educativa en los municipios analizados, tanto en promedio de años de escolaridad como en el porcentaje de población analfabeta en comparativa con el nivel nacional y estatal. 
TABLA 1

Panorama Educativo

\begin{tabular}{lll}
\hline & $\begin{array}{l}\text { Promedio de años de } \\
\text { escolaridad (años) }\end{array}$ & Porcentaje de población analfabeta (\%) \\
\hline Nacional & 9.2 & 5.5 \\
Durango & 9.1 & 3.2 \\
Santiago Papasquiaro & 8.4 & 2.5 \\
Tepehuanes & 7.3 & 2.2 \\
El Oro & 9.1 & 1.7 \\
\hline
\end{tabular}

Elaboración propia con datos de Instituto Nacional de Estadística y Geografía (2017)

\subsection{Educación a distancia}

La Asamblea General de las Naciones Unidas ha declarado el acceso a la Internet como un derecho humano por ser una herramienta que favorece el crecimiento y el progreso de la sociedad en su conjunto. El acceso a la Internet se ha vuelto una necesidad, pues en la educación, la salud, las empresas, incluso en el mismo hogar su uso es vital para el funcionamiento de un sinnúmero de procesos y actualmente en México es un derecho constitucional. En ese sentido, el avance y desarrollo de las TIC, pueden generar nuevas formas, estilos, tipos y procesos de educación, por medio del aprendizaje y la educación a distancia, Cabero y Martínez (2003). Desde la segunda mitad de la década de los 90, el vertiginoso crecimiento de las TIC ha obligado a los gobiernos de Latinoamérica a establecer políticas públicas desde la perspectiva de desarrollo de las TIC que se orientan a disminuir las brechas entre los países, así como al interior de ellos, y de esa manera promover la creación de sociedades de la información.

Aunado a lo anterior, determinar las necesidades de las comunidades en México es importante para diseñar nuevas ofertas educativas a distancia; que articule la educación, los empleadores y comunidad. Además, la educación a distancia contribuye en la sociedad a cambiar visiones, valores y dinámicas de aprendizaje con metodologías creativas e innovadoras, flexibilidad y adaptabilidad, valores y desarrollo de liderazgo, permitiendo una educación inclusiva (Castillo, 2015).

De esta manera, la educación a distancia contribuiría en la cobertura de la educación superior, la cual actualmente, resulta insuficiente. La inequidad en el acceso a la educación y el débil vínculo entre las empresas privadas y la educación pública son consecuencias de la deficiente educación en México. Es por ello, que en este caso la creación de nueva oferta en educación a distancia resulta relevante, ya que es una alternativa a la enseñanza tradicional en correspondencia con Gabotto y Castellanos (2015) y aumenta la cobertura de la UJED.

Los cambios en los paradigmas educativos han forzado a las universidades del mundo, a innovar, modificar o ampliar sus ofertas educativas y modalidades, como es el caso del Estado de Durango, donde sus dos principales ofertantes públicos de educación superior, han adoptado el sistema de educación a distancia, con el propósito principal de difundir conocimiento, de manera libre, a bajo costo y ampliar la cobertura en los municipios de la entidad. En el año 2009 se crea el Sistema Universidad Virtual, que abrió con seis campus y ofertando el mismo número de programas educativos. Actualmente, estas cifras iniciales se han incrementado, los campus ahora son doce y hay seis ofertas educativas diferentes, que pueden consultarse en la página oficial de la Universidad.

Respecto al acceso a recursos tecnológicos, se aprecia en la Tabla 2, el porcentaje de acceso a servicios tecnológicos en los tres municipios, los cuales muestran porcentajes muy bajos, de igual manera, en lo referente a la posesión de equipo de cómputo, pero no así con la probabilidad de poseer un celular. 
TABLA 2

Porcentajes de acceso tecnología

\begin{tabular}{llll}
\hline & $\begin{array}{l}\text { Acceso a Internet } \\
(\%)\end{array}$ & $\begin{array}{l}\text { Propiedad de un } \\
\text { celular }(\%)\end{array}$ & $\begin{array}{l}\text { Propiedad de una computadora } \\
(\%)\end{array}$ \\
\hline Santiago Papasquiaro & 11.1 & 70.4 & 28.7 \\
Tepehuanes & 7.52 & 61.9 & 35.77 \\
El Oro & 14.3 & 72 & 39.4 \\
\hline
\end{tabular}

Elaboración propia con datos del Instituto Nacional de Estadística y Geografía (2017)

\subsection{Perspectivas educativas}

El Diccionario de la Real Academia Española (Real Academia Española, 2014), define una perspectiva como el punto de vista desde el cual se considera o se analiza un asunto. En este estudio, dadas las fuentes de información y la forma en las que se consideran, se construye la siguiente definición de perspectiva educativa: El punto de vista desde el cual el agente informativo considera las preferencias, posibilidades y necesidades de opciones educativas de nivel superior. Es decir que una perspectiva no solo depende de un deseo, visión o supuesto personal, sino que se deriva del contexto familiar y social, pues como señalan Castillo, Mora y Téllez (2018), una consecuencia de las estrategias familiares es que los adolescentes pueden constituir estrategias personales y en consecuencia expectativas propias en él que, se encuentra el alumnado, sus padres y empleadores.

Por ello, la información proveniente del análisis de las perspectivas educativas, bajo la mirada de los empleadores que conocen las necesidades precisas de su región, así como las necesidades académicas permitirá, en el futuro, aumentar las posibilidades de conseguir un empleo tanto bien remunerado, como en su propio municipio, permitiendo paulatinamente el crecimiento económico. Por su parte, los padres de familia al aportar información de sus posibilidades económicas y su opinión con respecto a los estudios superiores de sus hijos y los deseos y necesidades de las y los alumnos sobre su educación, resultan relevantes para la UJED, para sustentar la generación de nueva oferta educativa en modalidad a distancia, así como deshabilitar o reestructurar las actuales.

\section{Procedimientos metodológicos}

La presente investigación es un estudio de caso, con un enfoque mixto, pues involucra información tanto cuantitativa como cualitativa en sentido transversal. La encuesta proporciona una descripción cuantitativa o numérica del comportamiento, del fenómeno de interés. (Creswell y Plano, 2007). El diseño de esta investigación fue no experimental porque los fenómenos fueron recabados en su contexto natural para ser analizados posteriormente y debido a que los datos se recogieron mediante la aplicación de tres instrumentos de medición, en un solo punto del tiempo, para cada municipio.

El enfoque cuantitativo se emplea en la recolección y el análisis de datos para medición numérica, con la posibilidad de establecer patrones de comportamiento en la población. Por otra parte, los aspectos cualitativos se generaron con las experiencias de los encuestadores en la fase de aplicación de los instrumentos, pero sobre todo con los foros organizados entre empleadores, que les permitió interactuar entre ellos de manera más abierta para expresar sus necesidades de preparación o formación académica tanto personal, como laboral para el crecimiento y desarrollo de su empresa y en el contexto de cada municipio. De manera que la investigación aporta datos interpretativos basados en las experiencias de campo. Se utilizó primero la inserción al campo para la recolección de descripciones, percepciones y observaciones de los participantes. 
Por el alcance de la investigación, se clasifica de tipo descriptivo, pues lo que se pretende es describir las características sociodemográficas, económicas y perspectivas educativas que se presentan en los hogares, estudiantes y empleadores que fueron sometidos al análisis.

\subsection{Equipo de trabajo}

El equipo de trabajo se formó en un inicio por la Coordinación General Académica integrada por tres investigadores de la UJED en el área de matemáticas aplicadas, trabajo social y economía. Además, de un equipo de apoyo local, tanto logístico como de contacto, integrado por los coordinadores del SUV en cada uno de los municipios, los cuales facilitaron los enlaces con las presidencias municipales y los directores de los centros educativos. Un tercer grupo fue conformado por estudiantes de servicio social y tesistas quienes fungieron como aplicadores, capturistas y colaboradores en el proceso de análisis de datos. En total el equipo conformado por 22 personas, lo cual fortaleció los aspectos importantes del estudio al generar un diagnóstico con resultados exploratorios, descriptivos y explicativos; para posibles nuevas ofertas educativas en modalidad a distancia de nivel superior.

\subsection{Lugar y población}

Durango es el cuarto estado de mayor extensión del país y gran parte de su territorio está formado por sierras y zonas montañosas, por lo que se dificulta acceder a algunos de los municipios. En esta primera etapa se decidió y autorizó por el PFCE iniciar con los municipios de Santiago Papasquiaro, Tepehuanes y El Oro; además se tomaron las cabeceras municipales porque se concentra el mayor número de escuelas de educación media superior de cada municipio.

La población objetivo fue el alumnado que en ese momento se encontraba estudiando el último año de educación media superior con una edad entre 16 y 18 años, sus padres de familia y los principales empleadores en cada uno de los tres municipios, se excluyeron estudiantes que no cursaban este año, o bien, en un centro educativo fuera de la cabecera municipal. La discriminación para padres de familia fue que no tuvieran hijos en los centros o año indicado. Para empleadores no hubo exclusión alguna, se tomaron en cuenta todas las opiniones de los empleadores que accedieron a responder el instrumento.

\subsection{Instrumentos}

Los autores Hernández, Fernández y Baptista (2008), validan que la selección de elementos depende de los criterios del investigador, por ello se generaron tres instrumentos diferentes para cada uno de los agentes informativos. El propósito fundamental de cada instrumento fue recopilar información para determinar un diagnóstico de perspectivas y necesidades de educación adecuada al contexto y municipios participantes en el estudio.

En una parte de los instrumentos se incluyeron aspectos de los estudios de pertinencia vigentes en la UJED, mientras que el resto de indicadores fueron diseñados con base en el interés propio de la investigación, pues este trabajo es pionero en su tipo al generar estudios de pertinencia contextualizados, multidisciplinarios y multifactoriales.

Los instrumentos fueron diseñados por la Coordinación General Académica:

1. El Cuestionario socioeconómico y de expectativas educativas de estudiantes de nivel medio superior está integrado por cinco secciones con 10 preguntas. A cada estudiante se le preguntó sobre sus planes a futuro y en caso de desear continuar estudiando, se procedió a cuestionarlos sobre lo que 
les gustaría estudiar y la modalidad e institución de estos. En este sentido, Mora (2014) valora la importancia de las perspectivas del alumnado para determinar los posibles factores que causan su deserción.

2. El Cuestionario socioeconómico y de expectativas educativas de las familias con hijos en nivel medio superior en municipios de Durango, dirigido a padres de familia o tutores del estudiantado. El cuestionario indaga en 26 preguntas, algunas características para cada uno de los integrantes del hogar, situación laboral, ingreso, economía familiar, calidad y espacios de la vivienda, aparatos tecnológicos, perspectiva y demanda educativa. El instrumento se adecuó con base en la Encuesta Nacional de Hogares de la Secretaría de Desarrollo Social (Encuesta Nacional de, 2014). Así mismo su perspectiva de que sus hijas e hijos continúen la educación superior.

3. El Cuestionario de expectativas educativas, para empleadores en municipios de Durango. Mediante este instrumento integrado con un total de 14 preguntas, los empleadores aportaron los datos de contacto de la empresa a la que representaban y sobre las necesidades particulares de formación académica de nivel superior que podrían influir en el crecimiento y desarrollo económico de la empresa a la que estaban representando. Adicionalmente, en el foro pudieron externar necesidades de capacitación y formación personal, empresarial y local.

Previa planeación con el Coordinador del Sistema Virtual en cada campus, se aplicó una prueba piloto para cada instrumento, durante la primera visita de reconocimiento; esto permitió precisar instrucciones y rediseñar la redacción de algunas preguntas. La versión final de los instrumentos se sometió al juicio de dos expertos uno de la Lock Haven University, Pensilvania, Estados Unidos, y otro de la Universidad de Texas en San Antonio, quienes aportaron ideas para la mejora del instrumento, alguna de ellas fue la recomendación de que a los padres de familia también se les preguntara acerca de su interés por seguir estudiando.

\subsection{Visitas de reconocimiento de los contextos de los tres municipios}

Durante el mes de junio y julio del 2017 se efectuaron visitas de reconocimiento de los contextos de los tres municipios. En la primera visita se aplicó la prueba piloto para los tres instrumentos, además se valoraron las condiciones generales de cada municipio para la aplicación final. En la segunda visita se explicó a los representantes de las presidencias municipales el objetivo del proyecto, se visitó a los directores de los centros educativos, se evaluó la conectividad a Internet y agendaron las fechas de aplicación. Como resultado del encuentro previo con directivos de los centros educativos se decidió aplicar un censo al alumnado de cada institución y se descartó el instrumento en línea por la baja calidad de la conectividad en los tres municipios.

\subsection{Capacitación de encuestadores y tesistas}

Derivado de que es una investigación interdisciplinaria, los encuestadores que participaron fueron acordes a la formación de los integrantes de la Coordinación General Académica; es decir de las facultades de Ciencias Exactas, Trabajo social y de Economía, Contaduría y Administración. Se registraron dos tesis de licenciatura una individual (Caballero, 2019) y otra conjunta con tres estudiantes (Castillo, et al., 2018), quienes también fungieron como encuestadores. Se elaboró el registró de las tesis y las asesorías se impartieron a lo largo de la ejecución del proyecto. Se concluyeron las dos tesis. La capacitación de los encuestadores se preparó antes la aplicación final. Se les explicó el propósito del proyecto, así como el contenido de cada uno de los instrumentos, se solventaron dudas y se les explicó la dinámica para la aplicación final. 


\subsection{Aplicación del instrumento}

La Coordinación General Académica junto con los encuestadores fueron los responsables de la aplicación de los instrumentos. Se desarrolló durante dos semanas del mes de agosto y septiembre del 2017. Para el alumnado fue en las instalaciones de su propia escuela, pues los directivos permitieron que la aplicación fuera en horas de clase, citaron a los padres de familia a una reunión con el propósito de dar respuesta al instrumento diseñado para ellos.

En los municipios de Santiago Papasquiaro y Tepehuanes se optó por ir a las direcciones de las empresas o negocios para la aplicación del instrumento. En El Oro se organizó un foro en la Casa de la Cultura de la cabecera; se contó con la asistencia de: docentes, comerciantes, empleados del ayuntamiento, agricultores, ganaderos y representantes de minas. A los empleadores se les aplicó el instrumento, seguido de un foro de opinión con la participación de todos los asistentes donde se obtuvo la información cualitativa sobre las necesidades de educación superior que consideraban podría impulsar el crecimiento económico y desarrollo social de su municipio, donde de manera abierta, conjunta y colaborativa expresaron sus opiniones sobre la temática de nueva oferta educativa pertinente a su contexto, la posibilidad económica que tienen los jóvenes para continuar con la educación superior.

\subsection{Gestión y tratamiento de la información}

Posterior a la aplicación del instrumento se procedió a impartir por parte de la Coordinación General Académica un taller de procesamiento estadístico a los encuestadores. En estos talleres se diseñaron las bases de datos en el paquete estadístico SPSS versión 15 para los tres instrumentos y se efectuaron los primeros resultados de estadística descriptiva. Después, la coordinación continuó el procesamiento de información. La parte cualitativa se trabajó con una matriz de categorías y las narrativas de los foros de opinión.

\section{ANÁLISIS Y DISCUSIÓN DE RESULTADOS}

A continuación, se detallan los principales hallazgos emanados de la investigación, a partir de los cuales se emiten una serie de conclusiones y recomendaciones que los convierte en la base para la futura toma de decisiones, tanto en la universidad, para la permanencia o rediseño de su actual oferta educativa, como para los ejecutores en los diferentes municipios evaluados. A continuación, se abordarán los resultados obtenidos de los tres instrumentos, desde los referentes teóricos de la investigación.

\subsection{Educación como potenciador de crecimiento económico y social}

Por parte de los empleadores, la gran mayoría corresponde al comercio con un 41.9\%, seguido por áreas gubernamentales y de la industria que representan actividades mineras y de la madera. Cabe mencionar que solo un bajo 3.2\% se registró en el área agrícola, debido posiblemente a que esta actividad se lleva a cabo de manera familiar y no en producción a gran escala.

Gabotto y Castellanos (2015) enfatizan en una de sus conclusiones que obtener un título universitario es más una presión social que una necesidad laboral, pues comentan que la persona egresada está consciente de que al llegar una oportunidad laboral lo que en realidad se valora serán las competencias logradas a lo largo de sus estudios, más que el estar titulado o no. Situación consistente con los resultados encontrados en el foro de empleadores. Además, ellos señalan que, en México, el trabajo es el que busca al empleado, y no al revés, es decir, las características de los puestos son fijas, y el empleado es quien tiene que ajustarse a esas necesidades. 
Ante este panorama poco alentador, la creación de oferta educativa en modalidad a distancia es pertinente, pues es un espacio de posibilidades para personas adultas que de verdad lo necesitan, previendo con ello menos posibilidad de deserción, pues es una alternativa, para alguien que posiblemente ya se encuentra en el mercado laboral y hará un esfuerzo consciente por terminar sus estudios.

Se encontró que los empleadores valoran mucho tanto el estar titulados como el tener experiencia laboral previa antes de comenzar a trabajar en la empresa. Considerando el apoyo que las empresas otorgan a sus empleados para ejecutar cursos en línea, los temas destacados de necesidad o interés expresados por los empleadores encuestados fueron temas relacionados con la administración en un 47.0\%, seguido por temas de contabilidad y mercadotecnia con una notable reducción de solo $16.0 \%$, por último, apoyo en idiomas y ventas son los últimos representativos con un $11.0 \%$ y $10.0 \%$ respectivamente.

En el Gráfico 1 se presentan las competencias que los empleadores destacan como imprescindibles en sus necesidades laborales, las cuales según su percepción podrían incrementar la productividad laboral y con ello derivar mayores ingresos para la empresa que laboran.

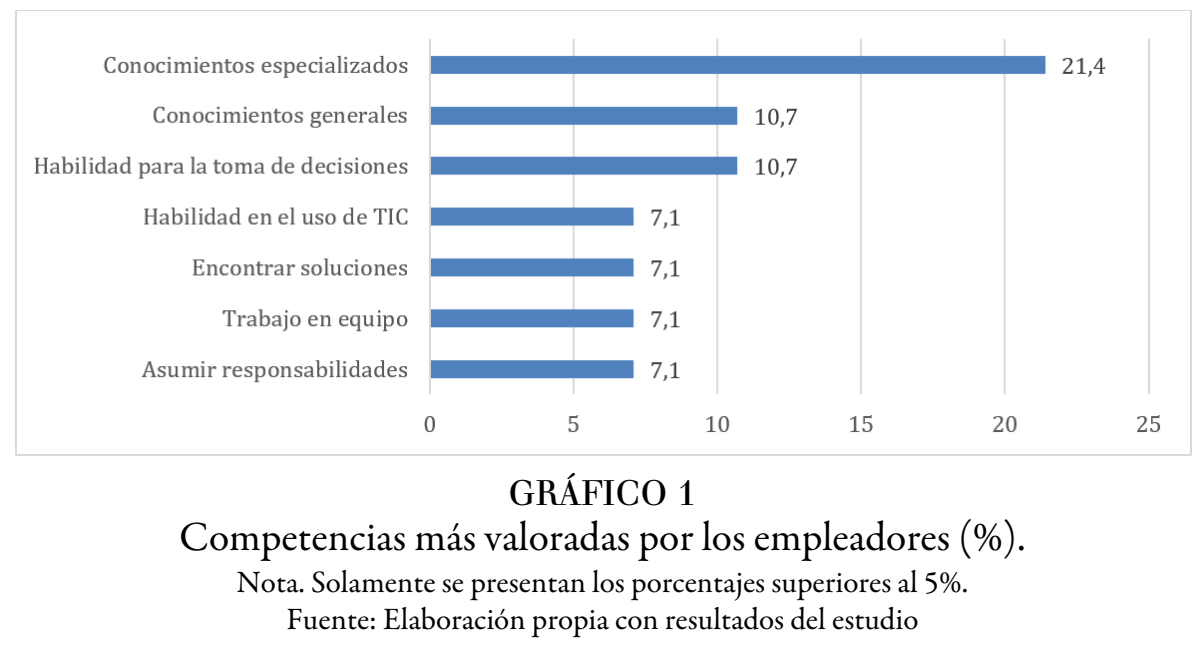

Por su parte entre las competencias menos valoradas son razonamiento lógico y analítico, la habilidad para buscar, procesar y analizar información de fuentes diversas, habilidad de dirección y coordinación, habilidad administrativa, capacidad para identificar, planear y resolver problemas, habilidad para las relaciones públicas, responsabilidad social y compromiso ciudadano y compromiso ético. Conocer estos resultados, ayudará en la planeación de las competencias que deben ser integradas o reforzadas en los procesos de rediseño o creación de nuevos planes de estudio en la universidad. Se destacan principalmente dos tipos de contratación que los empleadores pueden ofrecer a sus nuevos empleados con licenciatura, que son puestos de confianza con base y por contrato. Mientras que resulta poco común que los egresados sean subcontratados a través de una empresa externa.

Es notorio que el $48.4 \%$ de los empleadores expresa que la oferta actual puede cubrir sus necesidades mientras un $51.6 \%$ indica lo contrario. Esta disyuntiva de resultados es poco efectiva al momento de generar propuestas de desarrollo.

Las deficiencias más frecuentes expresadas por los empleadores respecto a sus empleados con licenciatura pueden apreciarse en el Gráfico 2. Es notoria la diversidad de resultados, lo cual puede ser atribuido a la propia diversidad de empleadores que participaron en el estudio y a sus necesidades particulares. El porcentaje de partición de empleadores por municipio fue de la siguiente manera, en Santiago Papasquiaro 39.0\%, en Tepehuanes 6.0\% y en El Oro de 55.0\%. 


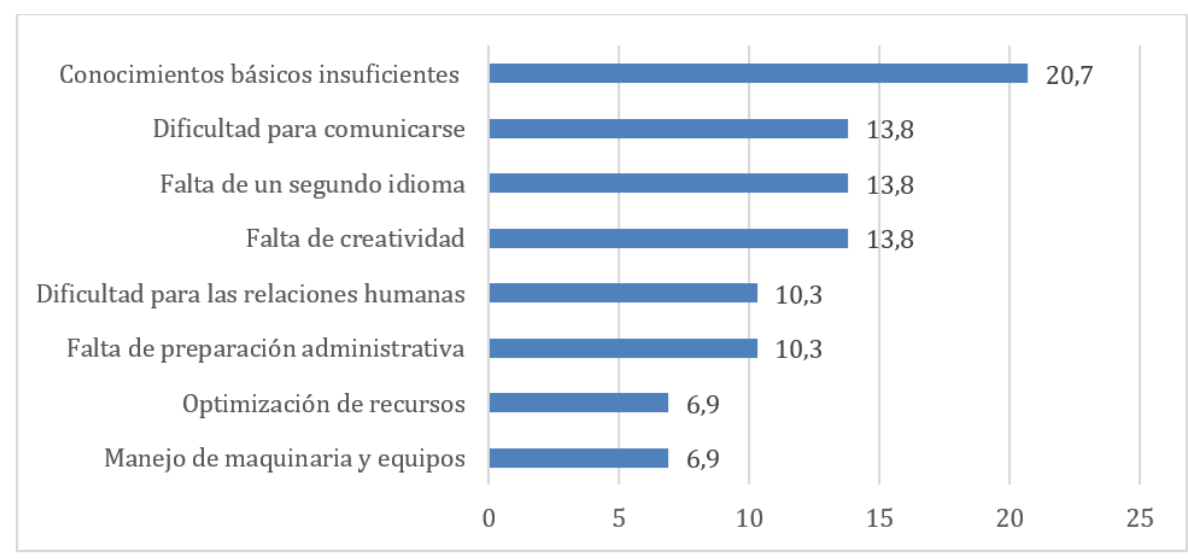

GRÁFICO 2

Deficiencias señaladas por los empleadores (\%).

Nota. Solamente se presentan los porcentajes superiores al $5 \%$. Elaboración propia con resultados del estudio

Las preferencias educativas expresadas por el estudiantado englobadas en áreas del conocimiento son las siguientes: el 24.0\% a las ingenierías enfocadas a minería, 20.0\% a Ciencias de la Salud y el 15.6\% a Ciencias Sociales y Administrativas, el 10.0\% en Ciencias Agropecuarias, puede observarse que estos resultados son consistentes con las características productivas de los municipios estudiados.

A pesar de que cada uno de los municipios evaluados cuenta con instalaciones físicas, estas carecen de calidad en la conectividad; actualmente la UJED no ofrece la mayoría de estos programas en modalidad a distancia, es por ello que los resultados encontrados cumplen con la necesidad de entregar un diagnóstico sobre las mejores opciones que pueden ofertarse y la necesidad de llevar a cabo campañas de difusión para esta modalidad.

\subsection{Educación como derecho social}

En esta investigación se contó con 572 estudiantes encuestados de educación media superior en el ciclo escolar 2017-2018. Santiago Papasquiaro cuenta con cuatro diferentes escuelas, seguido del municipio de El Oro con tres escuelas y una en Tepehuanes. Lo cual se muestra en los porcentajes de estudiantes $73.6 \%$ de Santiago Papasquiaro, 10.8\% Tepehuanes y $15.6 \%$ El Oro. El tipo de centro educativo que predomina en este nivel es el Colegio de Bachilleres. El 51.0\% de las y los estudiantes son hombres y el $49.0 \%$ mujeres.

El 40.0\% de los padres de familia encuestados tienen un nivel de escolaridad de secundaria completa, se les cuestionó sobre si ellos mismos estarían interesados en continuar con sus estudios; el 33.0\% de ellos expresó un interés en continuar sus estudios, sin embargo, el 82.0\% menciona no tener recursos para ello, al expresar como prioridad los estudios de sus hijos.

Puede apreciarse en el Gráfico 3, que más del 77.0\% de estudiantes expresan un deseo por continuar con estudios superiores, una vez que finalicen su actual nivel. En este mismo gráfico, se complementa con el lugar que estudiantes visualizan para continuar sus estudios. En Santiago Papasquiaro consideran permanecer en el municipio, lo cual resulta consistente debido a que se encuentra una Institución de educación superior. Por su parte, en Tepehuanes el estudiantado manifestó efectuar sus estudios en la capital del estado, puesto que no cuenta con una institución de educación superior en modalidad presencial en su municipio. Por último, estudiantes de El Oro expresan que estudiarían en otro estado debido a la cercanía que tienen con el estado de Chihuahua, a pesar de que ahí se ubica una institución de educación superior. 


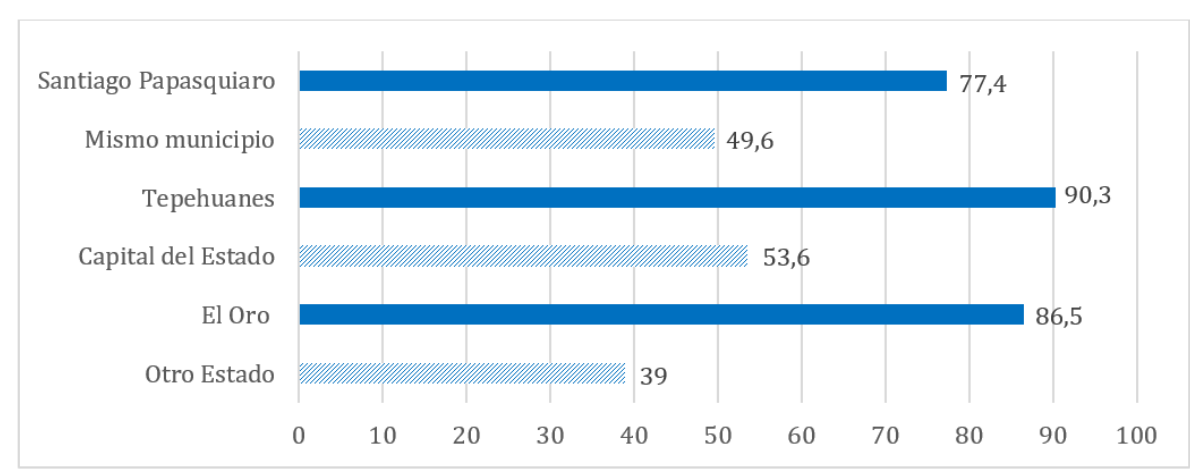

GRÁFICO 3

Porcentaje de alumnos que desea seguir estudiando y lugar de preferencia para hacerlo.

Nota: Se presenta únicamente los porcentajes más altos

Elaboración propia con resultados del estudio

\subsection{Educación a distancia}

A pesar de que en los tres municipios se localizan campus pertenecientes al SUV de la UJED. Del estudiantado encuestado, el 93.5\% de Santiago Papasquiaro, 96.7\% de Tepehuanes y 94.4\% de El Oro, manifestó no conocer la oferta educativa del SUV de la UJED. Dentro de las razones expresadas para no continuar en modalidad a distancia sus estudios superiores están: es mejor estar presente en las clases y la Internet no funciona en su municipio; esto acorde con Salgado y Rodríguez (2012) quienes destacan la importancia de la educación para reducir las condiciones de pobreza, sin embargo, señalan que la brecha de desigualdad educativa ha crecido. En estos contextos analizados la educación a distancia presenta barreras como lo es romper el paradigma de la educación tradicional. Los padres de familia también opinaron en su mayoría no conocer el SUV incluso ellos manifestaron estar interesados en continuar sus estudios (Castillo et al., 2018). Estos porcentajes llevan a reflexionar en cuanto a la difusión del campus, por parte de las autoridades de la UJED, en los tres municipios para que estudiantes y la comunidad estén informados de las opciones que tienen para continuar sus estudios.

Gómez y Cárdenas (2016) evalúan las perspectivas de esta modalidad tanto en docentes como en alumnos, encontrando que ambos coinciden en que la educación a distancia carece del sentido personal, pero no la descartan como modalidad de aprendizaje. Por su parte, los resultados mostraron que el $61.5 \%$ del estudiantado encuestados y ningún padre de familia visualiza la modalidad a distancia en la UJED para continuar sus estudios.

Sobresale el hecho de que solo el $19.4 \%$ de los empleadores tenga egresados de la UJED, pues se interpreta que los egresados de otras universidades están cubriendo esos puestos. Aunque el $71.4 \%$ de los empleadores indica que sabe de las dos modalidades tanto presencial como a distancia que ofrece la UJED, es preocupante que el otro $35.7 \%$ de ellos no conozca la oferta educativa, lo cual da un indicativo de que se requiere dar mayor difusión a las carreras ofertadas, resultados congruentes con los encontrados en padres de familia y alumnos quienes también señalaron el desconocimiento de la oferta.

\subsection{Perspectivas educativas}

La perspectiva del estudiantado es continuar sus estudios de educación superior la cual es consistente con el 88.0\% de sus padres que expresaron les gustaría que sus hijas e hijos continuaran estudiando el nivel superior, después de terminar la educación media. De manera global el $44.0 \%$ de los padres de familia, en Santiago Papasquiaro expresa que preferirían que sus hijos continuaran estudiando en el mismo municipio porque en 
la cabecera municipal ya existe una institución de educación superior. Mientras que los padres de familia en Tepehuanes expresan una movilidad a otro municipio o a la capital del Estado. Por su parte, los padres en El Oro opinan sobre la movilidad de sus hijos a otro estado, por la cercanía del estado de Chihuahua.

La inclinación del alumnado a estudios de minería concuerda con el contexto en el que viven, por ejemplo, en Santiago Papasquiaro se encuentran cuatro minas con extracción de oro, plata, plomo y zinc; mientras que en El Oro dos minas de oro, plata y fluorita (Servicio Geológico Mexicano, 2018). También el estudiantado valoró con un $20.0 \%$ el área de la salud, lo cual es consistente con el resultado de los foros organizados con los empleadores, en los cuales manifestaron la importancia de incrementar la formación de enfermeras y médicos (López, Luévano y Orrante, 2017).

\section{CONCLUSIONES Y RECOMENDACIONES}

El diagnóstico para nueva oferta educativa en modalidad a distancia derivado de los tres informantes, que fueron estudiantes del último año en nivel medio superior, sus padres o tutores y los empleadores de cada municipio evaluado, en conjunto expresan necesidades educativas de nivel superior, además de diplomados, cursos de profesionalización y educación continua, los cuales fueron englobados por áreas del conocimiento en orden de prioridad de la siguiente manera. En Ciencias de la Salud: Enfermería y Médicos; Ciencias Sociales: Trabajo Social; Tecnología: Logística y Computación; Ciencias Naturales y Exactas: Geoestadística, Geología, Minería; Agricultura: Tendencias innovadoras en agricultura y área forestal; Educación: Formación de profesores,

En la Tabla 3 se muestra un comparativo de las cinco primeras opciones de estudio, que expresaron estudiantes y empleadores. Puede observarse que las similitudes en las preferencias de las carreras, pero no en el orden de selección. El estudiantado opta por las ingenierías ofertadas actualmente en sus municipios, mientras que los empleadores aportan una opinión basada sus contextos laborales.

TABLA 3

Comparativo de las cinco primeras opciones de perspectivas educativas de estudiantes y empleadores

\begin{tabular}{lll}
\hline & Estudiantes & Empleadores \\
\hline $1^{\circ}$ opción & Ingenierias & Ciencias de la Salud \\
$2^{\circ}$ opción & Ciencias de la Salud & Ciencias Sociales y \\
& Ciencias Sociales y & Administrativas \\
$3^{\circ}$ opción & Administrativas & Tecnología
\end{tabular}

Elaboración propia con resultados del estudio

Por lo anteriormente expuesto, se cumplió con el diagnóstico para una nueva oferta educativa en modalidad a distancia para la UJED, además de que el estudio reveló otras áreas de investigación pertinentes y complementarias, como lo son la réplica de esta metodología en otros municipios que cuenten con instalaciones del SUV lo cual fortalecerá el diagnóstico a nivel estatal que posteriormente se puede llegar a replicar en otros estados del país. Cabe mencionar que en las visitas de reconocimiento y aplicación con los representantes gubernamentales, ellos solicitaron capacitación para participar en convocatorias gubernamentales en línea de proyectos productivos y obtener financiamientos extraordinarios así como capacitación para emprendedores.

En concordancia con Gabotto y Castellanos (2015) en lo referente a la dinámica cambiante de las necesidades laborales, los empleadores y sus subordinados expusieron la necesidad de tomar cursos cortos y puntuales respecto a temas específicos que les ayuden a mejorar su productividad, lo que a su vez es consistente con el aporte de Villafuerte y Benites (2018), que la educación y el conocimiento detonan crecimiento 
mediante la mejora en la mano de obra. Otra área de oportunidad encontrada fue que la propuesta de capacitación a distancia, no tiene que ser necesariamente de nivel superior, pues los resultados mostraron que hay interés de cursos breves, capacitación en áreas específicas, nivel medio superior y educación continua.

El segundo hallazgo importante, fue el descubrimiento de nuevos mercados interesados en la formación académica y capacitación continua, como lo son los padres de familia, que buscan opciones para concluir o avanzar con sus niveles educativos. La implementación de estas propuestas está fuera de las funciones del cuerpo de investigación. Con estos resultados y su difusión tanto a la Universidad, al gobierno del Estado y organismos nacionales e internacionales, se podrán establecer propuestas de acción para la consolidación de nueva oferta educativa con base en el diagnóstico encontrado. A pesar de haber logrado una propuesta para nueva oferta educativa en modalidad a distancia, esta solo podrá ser implementada al mejorar las condiciones de conectividad en los municipios y el acceso a servicios tecnológicos.

Además, es recomendable homogeneizar el tipo de respuesta de los tres instrumentos utilizados, para evaluar su validez y confiabilidad. Una última recomendación sugiere que esta metodología de estudios de caso se integre en los actuales estudios de pertinencia institucionales para el diseño y actualización de los planes y programas de estudio de la UJED. Para que esta institución pueda continuar a la vanguardia al ampliar sus ofertas educativas en la modalidad a distancia, deberá contextualizar sus planes y programas de estudio, sus campañas de difusión y de tal manera que su oferta educativa responda a las necesidades sociales y económicas del entorno, además es necesario que fortalezca y formalice vínculos con los diferentes actores que están presentes en la formación de estudiantes, como lo son la familia y el sector productivo.

\section{REFERENCIAS}

Bazdresch, M. (2001). Pobreza, desigualdad social y ciudadanía. Los límites de las políticas sociales en América Latina Pobreza y políticas sociales. En M. Bazdresch. (Ed.), Educación y pobreza: una relación conflictiva (pp. 65-81). Buenos Aires, Argentina: CLACSO. Recuperado de http://bibliotecavirtual.clacso.org.ar/clacso/gt/20101029 064158/6ziccardi.pdf

Caballero, A. (2019). Estudio Estadístico: expectativas educativas de los estudiantes del nivel medio superior, en tres municipios de Durango. (Tesis de Licenciatura). Universidad Juárez del Estado de Durango, México.

Cabero, J. y Martínez, F. (2003). Medio y Herramientas de Comunicación para la Educación Universitaria. Panamá: Sucesos Publicidad.

Castillo, C., (2015). Posicionando la educación inclusiva: Una forma diferente de mirar el horizonte educativo. Revista Educación, 39(2), 123-152. doi, https://doi.org/10.15517/REVEDU.V39I2.19902

Castillo, N., Mora, J., y Téllez, C., (2018). Caracterización sociodemográfica y perspectivas educativas de los padres de alumnos en educación media superior de tres municipios del Estado de Durango. (Tesis de Licenciatura). Universidad Juárez del Estado de Durango, México.

Creswell, J. y Plano, V. (2007). Designing and Conducting Mixed Methods Research. [El diseño y la realización de la investigación de métodos mixtos]. USA: Sage Publications.

Chaves, A., (1997). Economía y Educación. Revista Educación, 21(1), 97-107. doi: https://doi.org/10.15517/REVE DU.V21I1.7292

Consejo Nacional de Evaluación de la Política de Desarrollo Social, (2009). Metodología para la medición multidimensional de la pobreza en México. México: CONEVAL. Recuperado de https://bit.ly/2WfjIMK

Encuesta Nacional de Ingreso Gasto de los Hogares, (2014). Nueva Construcción. México: INEGI. Recuperado de h ttps://bit.ly/3dpUhxt

Gabotto, O. y Castellanos L. (2015). Panorama profesional en México, un escenario limitado para el estudiantado universitario. Revista Educación, 39(2), 115-122. doi, https://doi.org/10.15517/REVEDU.V39I2.19901.

Galeana, S. (2005). Promoción Social, Una opción metodológica. México: Plaza y Valdez Editores. 
Gómez, C. y Cárdenas, G. (2016). Distancia sin distancia: El oxímoron como lugar de encuentro de lo humano. Una mirada a la modalidad de educación a distancia desde la didáctica no parametral. Revista Educación, 40(2), 1-11. doi, https://doi.org/10.15517/REVEDU.V40I2.19488

Hernández, R., Fernández, C. y Baptista, P. (2008). Metodología de la Investigación. (4ºd.). México: Mc Graw Hill

Instituto Nacional de Estadística y Geografía, (2017), Anuario estadístico y geográfico de Durango 2017. Recuperado de https://datatur.sectur.gob.mx/ITxEF_Docs/DGO_ANUARIO_PDF.pdf

Instituto Nacional para la Evaluación de la Educación, (2011). La Educación Media Superior en México. Informe 2010-2011. México: INEE. Recuperado de https://www.inee.edu.mx/wp-content/uploads/2018/12/P1D235 .pdf

Kumar, A., Kumar, P., Palvia, S. y Verma, S. (2017) Online education worldwide: Current status and emerging trends. [Educación en línea en todo el mundo: Estado actual y tendencias emergentes].Journal of Information Technology Case and Application Research, 19(1), 3-9. doi, https://doi.org/10.1080/15228053.2017.1294867

López, A., Luévano K y., Orrante, M., (2017) Encuestas Hogares del Observatorio de Investigación Laboral. Manuscrito inédito. Durango, Universidad Juárez del Estado de Durango, México.

Márquez., A (2016). Editorial., La desigualdad social y las reformas educativas. En A. Márquez. (Ed.), Perfiles Educativos (pp. 3- 18). México: Instituto de Investigaciones sobre la Universidad y la Educación IISUE-UNAM. Recuperado de https://www.iisue.unam.mx/perfiles/articulo/2016-154.pdf

Mora A., (2014) La realización del derecho a la educación en América Latina: Análisis comparado de la eficiencia del gasto público y perspectivas educativas para la ciudad de Bogotá, Colombia. Revista Educación, 38(1),1-32. doi, https://doi.org/10.15517/REVEDU.V38I1.14375

Organización de las Naciones Unidas para la Educación, la Ciencia y la Cultura. (2012). Educación para el Desarrollo Sostenible. Francia: UNESDOC Biblioteca Digital. Recuperado de http://unesdoc.unesco.org/images/0021/0 02167/216756s.pdf

Organización de las Naciones Unidas para la Educación, la Ciencia y la Cultura, (2017). Educación para los Objetivos del Desarrollo Sostenible. Francia: UNESDOC Biblioteca Digital. Recuperado de https://bit.ly/2SLRzul

Real Academia Española, (2014). Diccionario de la lengua española, (23 a ed.). Madrid, España versión 23.3. Recuperado de https://dle.rae.es

Salgado J., y Rodríguez K. (2012). La desigualdad en Educación en México por Entidad Federativa 1995-2005. Revista Educación, 36(1), 45-62. doi, https://doi.org/10.15517/REVEDU.V36I1.451

Sánchez, C. y Ríos, H. (2011). La economía del conocimiento como base del crecimiento económico en México. Revista Venezolana de Información, Tecnología y Conocimiento, 8(2), 43-60. Recuperado de https://dialnet.unirioja.es/ servlet/articulo?codigo $=3703554$

Secretaría de Educación Pública (2012). Principales cifras del Sistema Educativo Nacional 2012-2013. México: Dirección General de Planeación, Programación y Estadística Educativa. Recuperado de https://bit.ly/3chapBw

Servicio Geológico Mexicano. (2018). Panorama Minero del Estado de Durango. Secretaría de Economía. México: Servicio Geológico Mexicano Recuperado de http://www.sgm.gob.mx/pdfs/DURANGO.pdf

Vasilevska, D., Rivza, B., Pivac, T., Alekneviciene, V. y Parlinska, A. (2017). Analysis of the Demand for Distance Education at Eastern and Central European Higher Education Institutions. Journal of Teacher Education for Sustainability. .(1), 106-116. doi, https://doi.org/10.1515/jtes-2017-0007

Villafuerte, J., y Benites, R., (2018), Competencias del profesional de la administración y finanzas para una economía basada en el conocimiento. Revista Educación, 42(2), 414-437. doi, https://doi.org/10.15517/REVEDU.V42I 2.27559

\section{BY-NC-ND}

